\title{
Guidance preconditioning by an impulse sequence for robust residual vibration suppression
}

\author{
I. Antoniadis \\ Machine Design and Control Systems Section, \\ Department of Mechanical Engineering, National \\ Technical University of Athens, P.O. Box 64078, \\ Athens 15710, Greece \\ E-mail:antogian@central.ntua.gr
}

Received 6 April 1998

Revised 5 May 1999

In order to suppress residual vibrations, a general method is presented for preconditioning any guidance function prior to its application to a dynamic system, by convolving it with a sequence of impulses. The approach includes first the development of the necessary design specifications for the impulse sequence, so that the robustness properties cover the widest possible variation of the system natural frequencies. Three solution methods are proposed then, with special emphasis in the achievement of the minimum possible duration time of the impulse sequence. Numerical experiments verify the effectiveness of the robustness, not only with respect to variations of the natural frequency, but also with respect to variations of a range of other linear and non-linear variables.

Keywords: Vibration suppression, vibration control, residual vibrations, guidance

\section{Introduction}

Residual vibration suppression is quite important in a broad range of mechanical engineering applications: space structures [5], cranes, textile machines, hydraulic machine tools [7], robot manipulators [1,6,8], computer disk heads [4], etc. The traditional approaches to minimize the effect of residual vibrations are focused either to a significant increase of the structure stiffness - resulting thus to bulky structures - or to the application of complex closed loop control methods, which in turn result to complex and failure liable motion systems.
An alternative approach to the above methods is the proper conditioning of the pre-specified excitation pattern ("Guidance"), so that the system moves exactly to the desired end position without any residual vibrations. This concept is very attractive, since it can significantly simplify the requirements of the motion equipment. Thus, significant research effort has been devoted towards this direction. The first approach historically introduced by Smith [17, p. 338], involves the partitioning of the desired excitation pattern into two distinct steps, the second one of which is delayed in time. Various other methods have been developed since then. Approaches generalizing the method proposed by Smith, include the introduction of multi-switch BangBang inputs [12], or the convolution of the desired guidance function with a series of impulses [13-16]. Other methods approximate the guidance function by a series of typical mathematical functions - such as splines [1,7,8], sinusoids [2], ramped sinusoids [10], or polynomials [4]. Closed form solutions are also available, using linear quadratic (LQ) optimal techniques [4].

The common feature of all the above methods is that they require the exact knowledge of the structure dynamic parameters (e.g., resonant frequencies, damping ratios, etc.). For this reason, their practical application has been quite limited, since even small variations of these parameters - due either to the poor modeling of the system or to changes of system variables during operation (e.g., machine payload) - cause significant deviations from the desired response and thus result to significant residual vibrations.

Requirements for the robust performance of the desired guidance function have been addressed relatively recently. The splines method proposed in $[7,8]$, is further extended in [1] with satisfactory results, covering also several possible "alternative dynamic models" of the system, which result from variations of its nominal parameters. However, the application of this method 
is limited only to systems where the desired guidance function can be approximated by splines, while the choice of the "alternative models" to be used in the design is significantly based on heuristic criteria.

In order to achieve a more robust behavior of the basic two equal impulse sequence [17, p. 338], the impulse sequence is extended to a specific pattern of a limited number of impulses (3 to 5) in several forms, like the ZVD(D) approach [13], or the Multi Hump EI approach [16]. Both the above approaches concern a SDOF system. In order to cover MDOF systems, the above basic impulse pattern is then further convolved [13] or delayed in time [14]. An alternative approach proposed [15], extends actually to MDOF systems the same basic set of equations of [13], using them either directly as a system of linear equations, or indirectly as constraints in a time minimization problem.

All the above methods still suffer from the fact that they require the knowledge of the system dynamic parameters, while they offer quite limited possibilities in the determination of the number of the necessary impulses, their duration and their magnitude. However, their major drawback is that the robustness they offer is restricted only in local areas around the system natural frequencies and can be increased only, by increasing the total duration of the pulse sequence. This results in unnecessary delays in the application of the total guidance and consequently, in the total duration of the system motion. Moreover, if the frequency ranges for the error minimization are not appropriately selected, significant error overshoots can result in other frequency areas.

This paper establishes the most general possible framework for preconditioning the desired guidance function by convolving it with an arbitrary sequence of impulses. In Sections 2-4, the necessary conditions for the robust behavior of the impulse sequence are first derived. Then, a general design approach is proposed, according to which the robustness properties cover now, not only local areas of variation around system natural frequencies, but the widest possible area for frequency variation. Finally, three methods are proposed for the design of the impulse sequence, with special emphasis in the achievement of the minimum possible duration of the impulse sequence. The first one is based on local design criteria, generalizing the approaches proposed in [13-16], while the other two are based on global error design criteria.

Section 5 includes a comparison of the already existing methods and the several options offered by the methods of this paper. Special emphasis is devoted to the possibilities of achieving the minimum possible duration time of the impulse sequence, while maintaining pre-specified error limits. Numerical experiments in Section 6 verify the effectiveness of the robustness, not only with respect to variations of the linear dynamic system parameters, but also with respect to variations of a range of non-linear coefficients and with respect to specific PID algorithms.

\section{Robustness requirements}

The equations of motion of a linear SDOF dynamic system with natural frequency $\omega$, damping ratio $\zeta$, excitation function (guidance) $u(t)$ and response vector $\mathbf{x}(t)$, can be written in the following state space form $[3,9]$ :

$$
\begin{aligned}
\dot{\mathbf{x}}(t) & =\mathbf{A} \mathbf{x}(t)+\mathbf{b} u(t), \\
\mathbf{A} & =\left[\begin{array}{cc}
0 & 1 \\
-\omega^{2} & -2 \zeta \omega
\end{array}\right], \quad \mathbf{b}=\left[\begin{array}{l}
0 \\
1
\end{array}\right] .
\end{aligned}
$$

Using the Duhamel integral, the system response is:

$$
\begin{aligned}
\mathbf{x}(t) & =\mathrm{e}^{\mathrm{A} t} \mathbf{x}(0)+\int_{0}^{t} \mathrm{e}^{\mathbf{A}(t-\tau)} \mathbf{b} u(t) \mathrm{d} \tau \\
& =\mathrm{e}^{\mathbf{A} t}\left[\mathbf{x}(0)+\int_{0}^{t} \mathrm{e}^{-\mathbf{A} \tau} \mathbf{b} u(t) \mathrm{d} \tau\right]
\end{aligned}
$$

The application of the Sylvester expansion method to the transition matrix $\mathrm{e}^{\mathbf{A} t}$ leads to:

$$
\begin{aligned}
\mathrm{e}^{\mathbf{A} t} & =\sum_{i=1}^{2} \mathrm{e}^{q_{i} t} \mathbf{H}_{i}, \\
q_{1,2} & =-\zeta \omega \pm \omega \sqrt{1-\zeta^{2}}, \\
\mathbf{H}_{i} & =\frac{1}{q_{i}-q_{j}}\left[\mathbf{A}-q_{i} \mathbf{I}\right] \\
& \text { for } i, j=1,2, i \neq j .
\end{aligned}
$$

Thus, substituting Eq. (3) in Eq. (2), the expression for the response can be written as:

$$
\begin{aligned}
\mathbf{x}(t) & =\mathrm{e}^{\mathbf{A} t}\left[\mathbf{x}(0)+\sum_{i=1}^{2} \mathbf{H}_{i} \mathbf{b} \int_{0}^{t} \mathrm{e}^{-q_{i} \tau} u(\tau) \mathrm{d} \tau\right] \\
& =\mathrm{e}^{\mathbf{A} t}\left[\mathbf{x}(0)+\sum_{i=1}^{2} \mathbf{H}_{i} \mathbf{b} U\left(s ; s=q_{i}\right)\right],
\end{aligned}
$$


where the integral in Eq. (4a) is easily recognized to be the Laplace transform $U(s)$ of the guidance function, evaluated at the two system poles $q_{i}$ in Eq. (3b). In case of a rigid body motion $(\omega=0)$, Eq. (4b) can be similarly shown to take the specific form:

$$
\begin{aligned}
\mathbf{x}_{\mathrm{R}}(t) & =\mathbf{H}_{\mathrm{R}}(t)[\mathbf{x}(0)+\mathbf{b} U(s ; s=0)], \\
\mathbf{H}_{\mathrm{R}}(t) & =\left[\begin{array}{ll}
1 & t \\
0 & 1
\end{array}\right] .
\end{aligned}
$$

The preconditioning method proposed, involves the convolution of the "original guidance function" $g(t)$ with a series of impulses $p(t)$ :

$$
\begin{aligned}
& u(t)=\int_{0}^{t} g(t-\tau) p(\tau) \mathrm{d} \tau \\
& p(t)=\sum_{n=0}^{N} c_{n} \delta(t-n T),
\end{aligned}
$$

were $\delta(t)$ is the delta (Dirac) impulse function. Thus, the implementation of Eq. (6a) in practice only requires that:

$$
u(t)=\sum_{n=0}^{N} c_{n} g(t-n T) .
$$

The Laplace transform of Eq. (6) is:

$$
\begin{aligned}
& U(s)=G(s) P(s), \\
& P(s)=\sum_{n=0}^{N} c_{n} \mathrm{e}^{-s n T} .
\end{aligned}
$$

The purpose of introducing the impulse sequence $p(t)$, is that the conditioned guidance function $u(t)$ is able to move the system in essentially the same way as the original guidance function $g(t)$, without the effect of the residual vibrations. This requirement can be stated as:

$$
\begin{gathered}
P\left(s ; s=q_{i}\right)=0, \\
P(s ; s=0)=1 .
\end{gathered}
$$

In view of the combination of Eqs (3b), (4b), (7a), Eq. (8a) implies that the residual vibration effect is completely canceled. In view of Eq. (7a), this can be accomplished just by the proper design of only the sequence $p(t)$, quite independently from the properties of the original guidance function $g(t)$. Parallel, in view of the combination of Eqs (5) and (7a), Eq. (8b) states that the pulse sequence $p(t)$ maintains all the properties that $g(t)$ posses, in order to move the system as a rigid body. Thus, Eq. (8) are the necessary conditions for $p(t)$, in order to bring the system to the desired end position, without the effect of residual vibrations. Substitution of Eq. (3b) in (8) leads to:

$$
\begin{aligned}
& \sum_{n=0}^{N} c_{n} \mathrm{e}^{n \zeta \omega T} \cos \left(n \omega T \sqrt{1-\zeta^{2}}\right)=0, \\
& \sum_{n=0}^{N} c_{n} \mathrm{e}^{n \zeta \omega T} \sin \left(n \omega T \sqrt{1-\zeta^{2}}\right)=0, \\
& \sum_{n=0}^{N} c_{n}=1 .
\end{aligned}
$$

The effect of the damping coefficient $\zeta$ in the system response is two-fold. First, it has a positive effect, resulting in the damping of the residual vibrations. Second, it produces a shift in the natural frequency of the undamped system by a factor of $\sqrt{1-\zeta^{2}} N$. Thus, provided that the design of the sequence $p(t)$ is robust enough to cover that amount of system natural frequency variations, damping can be neglected and Eq. (9) can be simplified as follows, without any loss in the generality of application:

$$
\begin{aligned}
& \sum_{n=0}^{N} c_{n} \cos (n \phi)=0, \\
& \sum_{n=0}^{N} c_{n} \sin (n \phi)=0, \\
& \sum_{n=0}^{N} c_{n}=1, \\
& \phi=\omega T .
\end{aligned}
$$

Further treatment of Eqs (10a), (10b) lead to two equivalent sets of equations, depending on the number of coefficients $N$.

\section{Case I ( $N$ even)}

$$
\begin{aligned}
& a_{0} / 2+\sum_{l=1}^{L} a_{l} \cos (l \phi)=0, \\
& \sum_{l=1}^{L} b_{l} \sin (l \phi)=0,
\end{aligned}
$$




$$
L=N / 2 \text {. }
$$

where the impulse sequence coefficients $c_{n}$ can be determined as follows:

$$
\begin{array}{ll}
c_{j}=\left(a_{L-j}+b_{L-j}\right) / 2, & j=0, L-1, \\
c_{L}=a_{0} / 2, & \\
c_{j}=\left(a_{j-L}+b_{j-L}\right) / 2, & j=L+1, N .
\end{array}
$$

Case II ( $N$ odd $)$

$$
\begin{aligned}
& \sum_{l=1}^{M} a_{l} \cos \left(\frac{2 l+1}{2} \phi\right)=0, \\
& \sum_{l=1}^{L} b_{l} \sin \left(\frac{2 l+1}{2} \phi\right)=0, \\
& M=(N-1) / 2
\end{aligned}
$$

where the impulse sequence coefficients $c_{n}$ can be determined as follows:

$$
\begin{aligned}
& c_{j}=\left(a_{M-j}+b_{M-j}\right) / 2, \quad j=0, M, \\
& c_{j}=\left(a_{j-M-1}+b_{j-M-1}\right) / 2, \quad j=M+1, N .
\end{aligned}
$$

In both cases, an obvious and convenient choice for the coefficients $b_{i}$ is:

$$
b_{i}=0
$$

Thus Eqs (11b) and (13b) are identically satisfied, the number of the unknown coefficients is reduced by half according to Eqs (11a), (13a) and the coefficients $c_{n}$ are now symmetric around $N / 2$ according to Eqs (12) and (14).

\section{Design specifications}

In view of Eq. (15), Eqs (11) and (10c) now become:

$$
\begin{aligned}
& W_{a \mathrm{I}}(\omega)=a_{0} / 2+\sum_{l=1}^{L} a_{l} \cos (l \omega T)=0, \\
& \omega \neq 0, \\
& W_{a \mathrm{I}}(0)=a_{0} / 2+\sum_{l=1}^{L} a_{1}=1 .
\end{aligned}
$$

Similarly, Eqs (13) and (10c) become:

$$
\begin{aligned}
& W_{a \mathrm{II}}(\omega)=\sum_{l=0}^{M} a_{l} \cos \left(\frac{2 l+1}{2} \omega T\right)=0, \\
& \omega \neq 0, \\
& W_{a \mathrm{II}}(0)=\sum_{l=0}^{M} a_{1}=1 .
\end{aligned}
$$

Eqs (16) and (17) satisfy the following symmetry properties:

$$
\begin{aligned}
& W_{a \mathrm{I}}(\phi+2 \pi)=W_{a \mathrm{I}}(\phi), \\
& W_{a \mathrm{I}}(2 \pi-\phi)=W_{a \mathrm{I}}(\phi), \\
& W_{a \mathrm{II}}(\phi+2 \pi)=-W_{a \mathrm{II}}(\phi), \\
& W_{a \mathrm{II}}(2 \pi-\phi)=-W_{a \mathrm{II}}(\phi), \\
& W_{a \mathrm{II}}(\phi+4 \pi)=W_{a \mathrm{II}}(\phi), \\
& W_{a \mathrm{II}}(4 \pi-\phi)=W_{a \mathrm{II}}(\phi) .
\end{aligned}
$$

Thus, Eqs (16) and (17) define a residual vibration error function $W_{a}(\omega)$, dependent on the system eigenfrequency $\omega$. In order that the pulse sequence $p(t)$ is robust with respect to the variations of the system natural frequency $\omega$, the error function $W_{a}(\omega)$ must be zero for all the possible values of $\omega$, except the rigid body motion case of $\omega=0$. Figure 1 presents the ideal behavior $W_{D}(\omega)$ of this error function. The form of $W_{D}(\omega)$ is constructed in such a way, that for a small frequency area $B$ near $\omega=0$ the "rigid body properties" of $g(t)$ are retained, while residual vibrations are suppressed over eigenfrequency values above $\omega_{c}$. Thus, a design problem can be now formulated: The parameters of the function $W_{a}(\omega ; a, N, T)$ must be determined in such a way, that $W_{a}(\omega)$ approximates 'as close as possible' the ideal behavior of $W_{D}(\omega)$.

In view of the symmetry properties (18) and (19), the maximum design frequency achievable in Fig. 1 is determined by:

$$
\begin{aligned}
& \omega_{\max }=\pi / T=N \pi / T_{p}=N \omega_{p} / 2, \\
& \omega_{\text {rep }}=2 \omega_{\max }, \\
& T_{p}=N T, \\
& \omega_{p}=2 \pi / T_{p},
\end{aligned}
$$

where $T_{p}$ is the total duration time of the impulse sequence $p(t)$ and $\omega_{p}$ the characteristic impulse sequence frequency. 


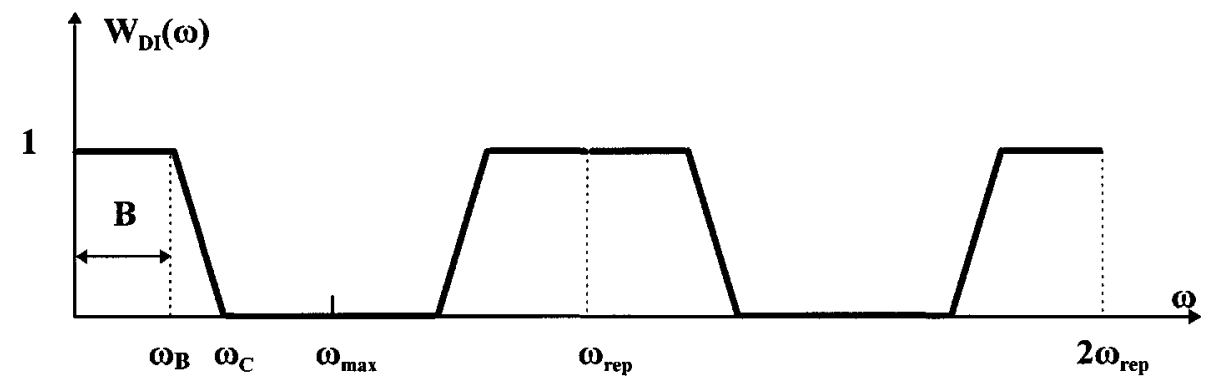

(a)

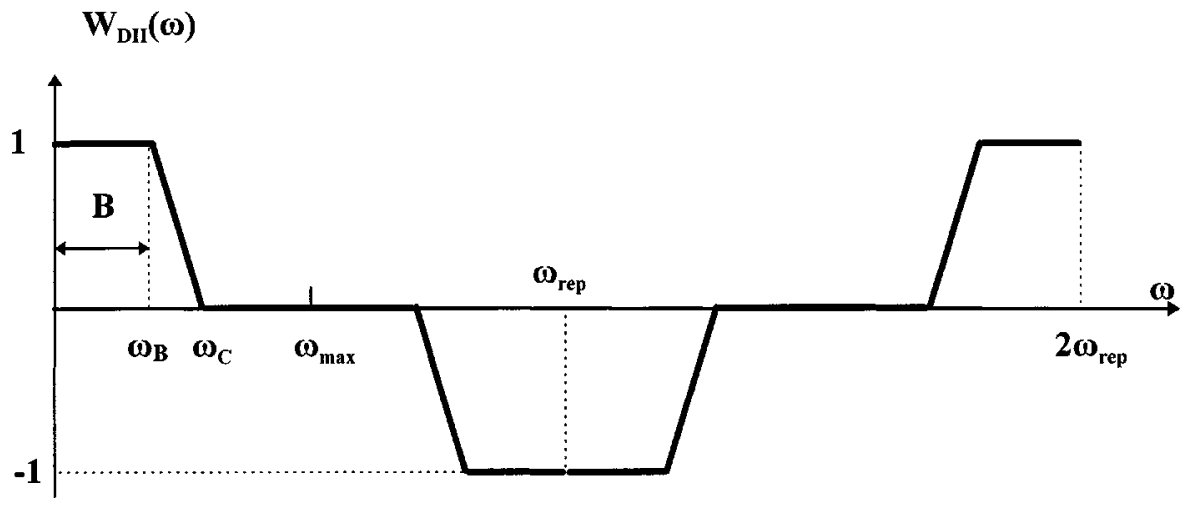

(b)

Fig. 1. Ideal behavior of a residual vibration error function. (a) Case I, (b) Case II.

\section{Design methods}

Currently, a large number of methods [11] are available for the approximation of $W_{D}(\omega)$ by Eqs (16), (17). Proper adaptation of them leads - among others - to three interesting solution approaches.

\subsection{Direct solution approach}

According to this approach, the function $W_{a}(\omega)$ takes exactly the values of $W_{D}(\omega)$ in a set of $L$ or $M$ distinct frequencies $\omega$. This leads to a system of equations in the form of:

\section{Case I}

$$
\begin{gathered}
{\left[\begin{array}{cccc}
\cos \left(\phi_{1}\right) & \cos \left(2 \phi_{1}\right) & \cdots & \cos \left(L \phi_{1}\right) \\
\cos \left(\phi_{2}\right) & \cos \left(2 \phi_{2}\right) & \cdots & \cos \left(L \phi_{2}\right) \\
\ldots & \cdots & \cdots & \cdots \\
\cos \left(\phi_{L}\right) & \cos \left(2 \phi_{L}\right) & \cdots & \cos \left(L \phi_{L}\right)
\end{array}\right]} \\
\times\left[\begin{array}{c}
a_{1} \\
a_{2} \\
\ldots \\
a_{L}
\end{array}\right]=\left[\begin{array}{c}
1 \\
1 \\
\cdots \\
1
\end{array}\right]\left(-a_{0} / 2\right)
\end{gathered}
$$

completed by Eq. (16b) after an initial choice of $a_{0}$. The elements of the matrix in the left hand side of Eq. (21a) can be defined as:

$$
\begin{aligned}
{[\Phi]_{i j} } & =\cos \left(j \phi_{i}\right)=T_{j}\left(y_{i}\right), \\
y_{i} & =\cos \left(\phi_{i}\right),
\end{aligned}
$$

where $T_{l}(y)$ is an $l$-th order Chebyshev polynomial of $y[11]$.

\section{Case II}

$$
\left[\begin{array}{cccc}
\cos \left(3 \frac{\phi_{1}}{2}\right) & \cos \left(5 \frac{\phi_{1}}{2}\right) & \cdots & \cos \left[(2 M+1) \frac{\phi_{1}}{2}\right] \\
\cos \left(3 \frac{\phi_{2}}{2}\right) & \cos \left(5 \frac{\phi_{2}}{2}\right) & \cdots & \cos \left[(2 M+1) \frac{\phi_{2}}{2}\right] \\
\cdots & \cdots & \cdots & \cdots \\
\cos \left(3 \frac{\phi_{M}}{2}\right) & \cos \left(5 \frac{\phi_{M}}{2}\right) & \cdots & \cos \left[(2 M+1) \frac{\phi_{M}}{2}\right]
\end{array}\right]
$$




$$
\times\left[\begin{array}{c}
a_{1} \\
a_{2} \\
\cdots \\
a_{L}
\end{array}\right]=\left[\begin{array}{c}
\cos \left(\frac{\phi_{1}}{2}\right) \\
\cos \left(\frac{\phi_{2}}{2}\right) \\
\cos \left(\frac{\phi_{M}}{2}\right)
\end{array}\right]\left(-a_{0}\right)
$$

completed by Eq. (17b) after an initial choice of $a_{0}$. The elements of the matrix in the left hand side of Eq. (22a) can be defined as:

$$
\begin{aligned}
{[\Phi]_{i j} } & =\cos \left[(2 j+1) \varphi_{i} / 2\right]=T_{2 j+1}\left(y_{i}\right), \\
y_{i} & =\cos \left(\phi_{i} / 2\right)
\end{aligned}
$$

Using the classical properties of determinants, it can be easily shown that the determinant of $\Phi$ takes the form of a Vandermonde determinant, the columns of which are polynomials of $y_{l}$. Provided that the values of $\varphi$ in Eqs (21a) and (22a) are different from each other and they do not result in zero cosine terms, this determinant is different than zero and thus, the systems in Eqs (21a), (22a) can lead to a nontrivial solution.

In fact, practical applications of this method or its slight variations, already exist in the literature. The "alternative modeling approach", proposed in [1] for the specific case of spline functions, is based on this concept. Also, by selecting a set of individual frequencies $\omega_{i}$ quite close to each other, robustness issues can be locally added, leading in practice to the same results as in $[13,15]$. In case that the error is not requested at a specific frequency to be exactly zero, but rather equal to a small percentage (e.g., 5\%), an additional right hand vector can be added to Eqs (21a) and (22a), leading to the same results as in [16].

Although the method has excellent localization properties for specific values of $\omega$, it suffers from numerical instability problems, inherent in systems of the Vandermonde structure, especially for a large number of unknowns. This results among others to values of the error function $W_{a}(\omega)$ significantly exceeding unity at specific frequencies, not explicitly introduced in Eqs (21), (22). Increasing the number of equations in Eqs (21a), (22a) by introducing additional frequencies not appropriately selected, leads to the aggravation of the above situation.

In order to overcome this disadvantage, two other methods can be used alternatively, based now on global approximation criteria dependent on the general shape of the function $W_{D}(\omega)$.

\subsection{Fourier approximation}

Since $W_{D}(\omega)$ must also satisfy the symmetry and periodicity properties of Eqs (18), (19), Fourier series can be used for its approximation by the direct calculation of the coefficients $a_{k}$. Thus:

Case I

$a_{k}=\frac{2}{\pi} \int_{0}^{\pi} W_{D \mathrm{I}}(\phi) \cos (k \phi) \mathrm{d} \phi, \quad k=0, L$.

In the general case, the fast Fourier transform (FFT) can be used for the evaluation of Eq. (23). In the specific case that $W_{a}(\omega)$ takes the analytical form of Fig. 1(a), a closed form solution exists for Eq. (23):

$$
\begin{aligned}
a_{0}= & \left(\phi_{\mathrm{B}}+\phi_{\mathrm{C}}\right) / \pi, \\
a_{k}= & \frac{4}{\pi k^{2}\left(\phi_{\mathrm{C}}-\phi_{\mathrm{B}}\right)} \sin k \frac{\phi_{\mathrm{C}}+\phi_{\mathrm{B}}}{2} \\
& \times \sin k \frac{\phi_{\mathrm{C}}-\phi_{\mathrm{B}}}{2}, \quad k=1, L .
\end{aligned}
$$

Case II

$$
\begin{aligned}
a_{k} & =\frac{2}{\pi} \int_{0}^{\pi} W_{D \mathrm{II}}(\phi) \cos [(2 k+1) \phi / 2] \mathrm{d} \phi, \\
k & =0, M .
\end{aligned}
$$

Similarly, in the specific case that $W_{D}(\omega)$ takes the analytical form of Fig. 1(b), a closed form solution exists for Eq. (25):

$$
\begin{aligned}
a_{k}= & \frac{4}{\pi[(2 k+1) / 2]^{2}\left(\phi_{\mathrm{C}}-\phi_{\mathrm{B}}\right)} \sin \frac{2 k+1}{2} \frac{\phi_{\mathrm{C}}+\phi_{\mathrm{B}}}{2} \\
& \times \sin \frac{2 k+1}{2} \frac{\phi_{\mathrm{C}}-\phi_{\mathrm{B}}}{2}, \quad k=0, M
\end{aligned}
$$

\subsection{Chebyshev approximation}

\section{Case I}

Defining $y$ as:

$$
y=\cos (\varphi)
$$

$W_{D \mathrm{I}}(\omega)$ can be approximated by a Chebyshev series of the form [11]:

$$
W_{D \mathrm{I}}(y) \approx \frac{a_{0}}{2}+\sum_{k=1}^{L} a_{k} T_{k}(y) .
$$


The solution is

$a_{k}=\frac{2}{L+1} \sum_{r=0}^{L} W_{D \mathrm{I}}\left(\phi_{r}\right) T_{k}\left(y_{r}\right), \quad k=0, L$,

where $y_{r}\left(\phi_{r}\right)$ are the $L+1$ roots of the $T_{L+1}$ polynomial:

$$
\begin{aligned}
& T_{L+1}\left(y_{r}\right)=0, \\
& y_{r}=\cos \left(\phi_{r}\right), \\
& \phi_{r}=\frac{2 r+1}{2(L+1)} \pi,
\end{aligned}
$$

where $r=0, L$.

Thus Eq. (17c) becomes

$a_{k}=\frac{2}{L+1} \sum_{r=0}^{L} W_{D \mathrm{I}}\left(\phi_{r}\right) \cos \left(k \phi_{r}\right), \quad k=0, L$.

\section{Case II}

Similarly, defining

$$
y=\cos (\theta)=\cos (\varphi / 2)
$$

the following solution can be shown to exist for the coefficients $a_{k}$ :

$$
\begin{aligned}
a_{k} & =\frac{2}{M+1} \sum_{r=0}^{M} W_{D \mathrm{II}}\left(\phi_{r}\right) \cos \left(\frac{2 k+1}{2} \phi_{r}\right) \\
k & =0, M \\
\phi_{r} & =\frac{2 r+1}{2(M+1)} \pi .
\end{aligned}
$$

The Chebyshev polynomial is known to be [11] quite close to the minmax polynomial, best approximating the original function. It typically converges for a small number $L$ or $M$ of coefficients, compared to other polynomial approximating methods.

\section{Comparison of design methods}

Figure 2 presents a comparison of the results of the Two Hump Extra Insensitive input shaper developed in [16] to the Fourier method (Case I) developed in this paper. In both methods, the total pulse duration time $T_{p}$ is chosen equal to 1.5 times the system eigenperiod, corresponding to an eigenfrequency of $1 \mathrm{rad} / \mathrm{s}$.

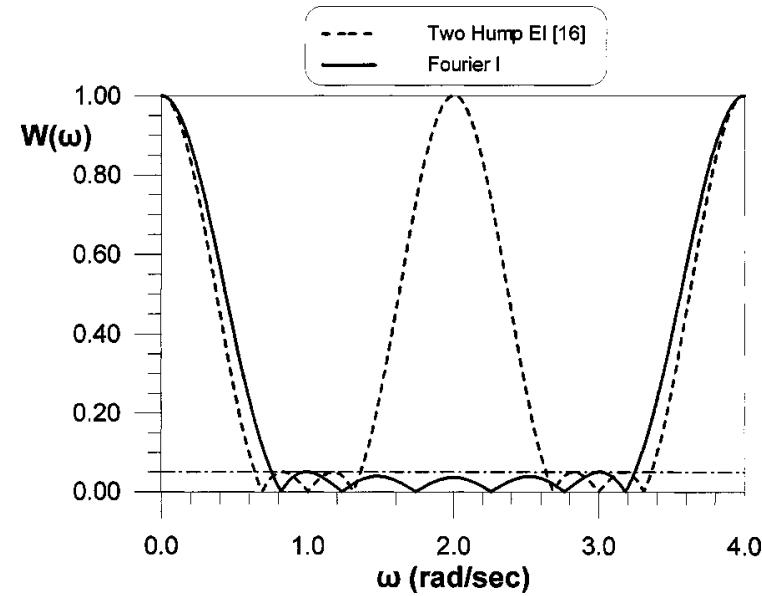

Fig. 2. Comparison of the proposed methods to the multi-hump EI input shapers method [16].

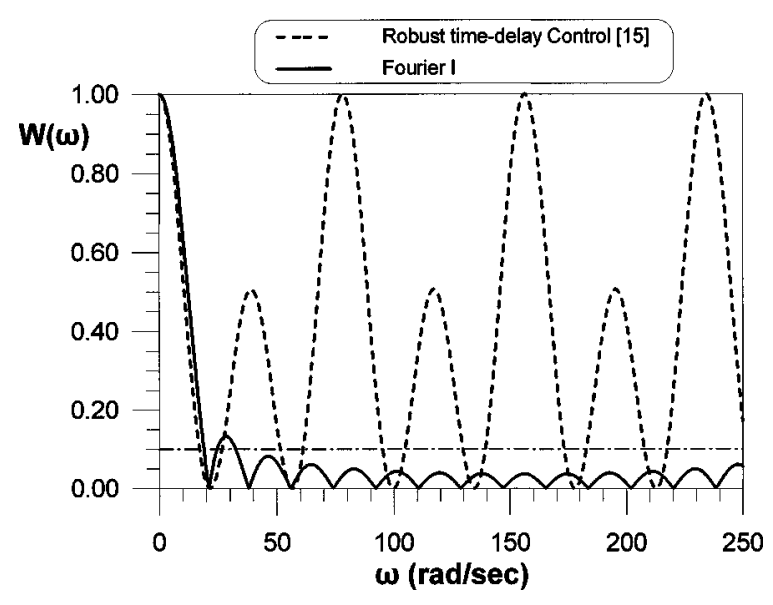

Fig. 3. Comparison of the proposed methods to the robust time-delay control method [15].

The number of pulses used is 4 for the Two Hump EI method and 7 for the Fourier method. The Fourier method is able to eliminate the central lobe appearing around $2 \mathrm{rad} / \mathrm{s}$.

Figure 3 presents a comparison of the results of the minimum time robust design, proposed in [15] to the Fourier method (Case I) developed in this paper. The example concerns a $2 \mathrm{DOF}$ system with two modes at $21.6 \mathrm{rad} / \mathrm{s}$ and $212.59 \mathrm{rad} / \mathrm{s}$. The error curve presenting the results of [15] was reproduced by the Direct Solution approach, using two points, each one quite close to the above 2 frequencies. In both methods, the total pulse duration time $T_{p}$ is chosen equal to $0.3216 \mathrm{~s}$. The number of pulses used is 9 for the method of [15] and 17 for the Fourier method. The Fourier method is able 


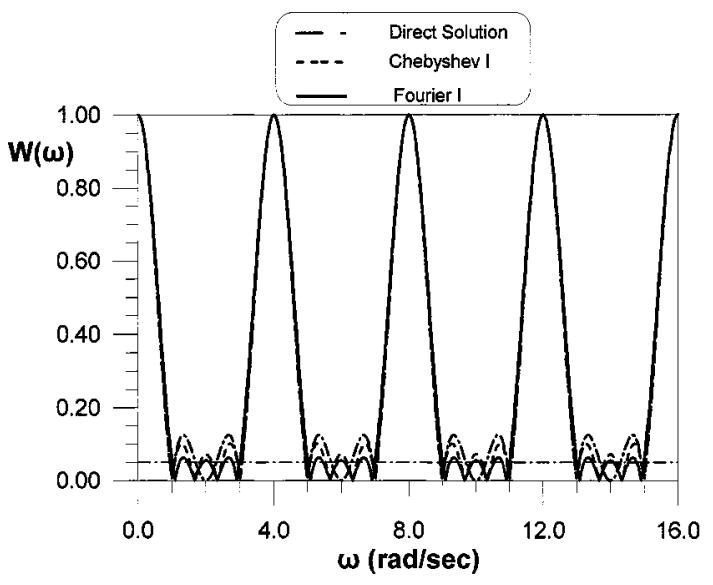

(a)

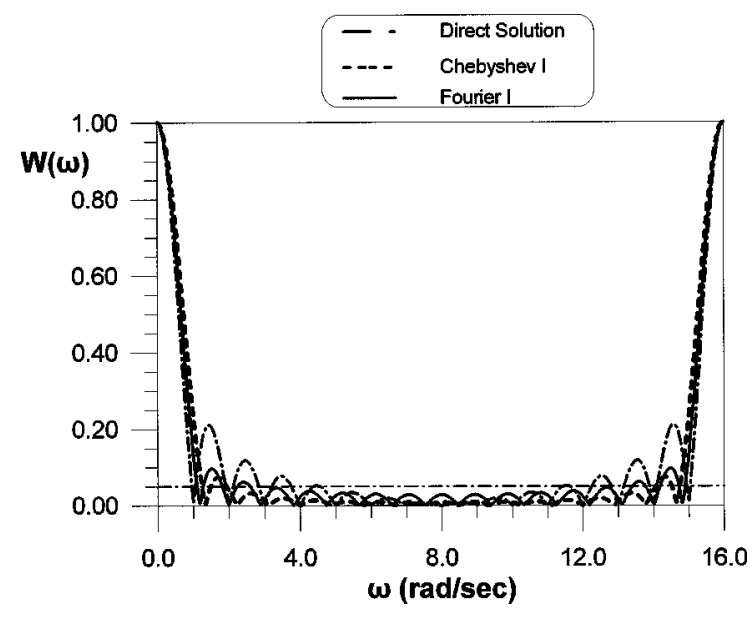

(b)

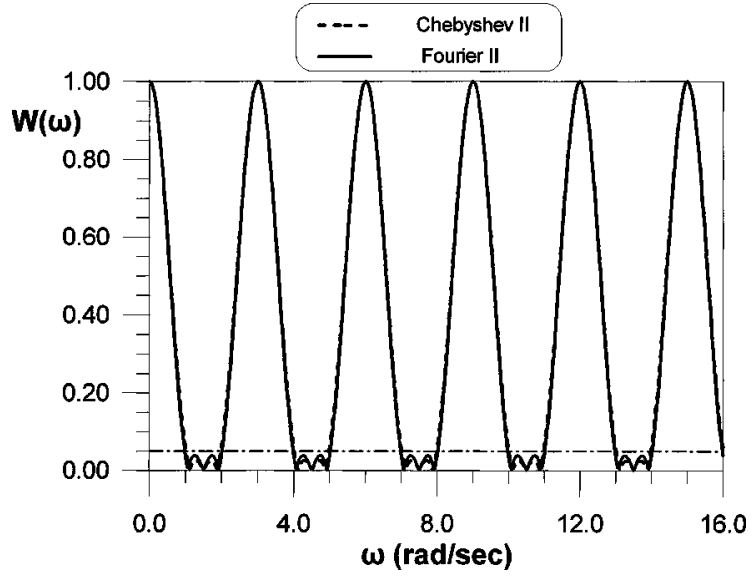

(c)

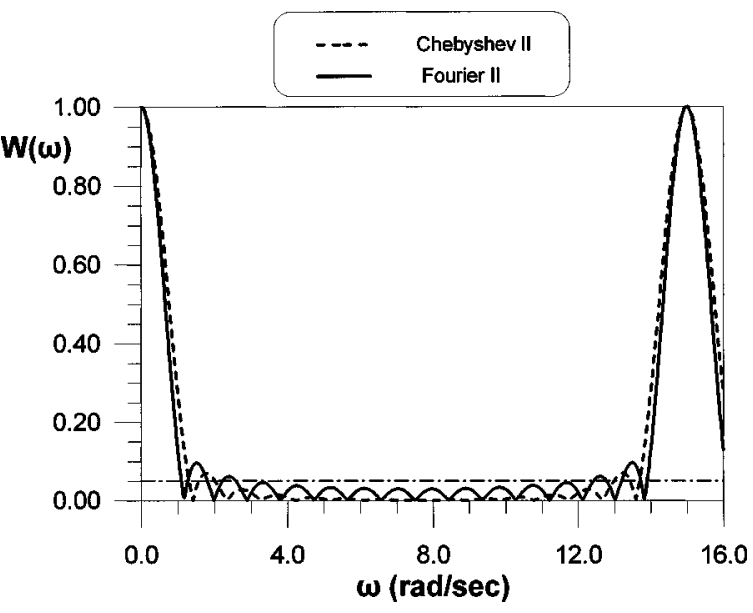

(d)

Fig. 4. Error functions of the proposed methods for $T_{p}=2 \pi$. (a) $N=4$ (Case I), (b) $N=16$ (Case I), (c) $N=3$ (Case II), (d) $N=15$ (Case II).

to eliminate the residual vibration error in the whole range between 20 and $250 \mathrm{rad} / \mathrm{s}$.

Both the above figures clearly show the problems associated with the alternative approaches in the literature. Robustness properties are improved only in a close area around the specified frequency. Significant residual vibration errors remain in the rest of the frequency area. Contrary, the methods proposed in this paper indicate that by properly increasing the number of pulses and designing them according to global criteria, the robust frequency area can be drastically increased.

Then, the effect of increasing the number of pulses in the proposed methods is examined. Figure 4 presents results for all the proposed methods, when the num- ber of pulses is increased practically by 4 , without increasing the total pulse duration time. For the Direct Solution Approach, the necessary frequency values in Eq. (21a) are equally spaced in the range $\left[\omega_{\mathrm{C}}, \omega_{\max }\right]$. This appears to be the best way to use the Direct Solution Approach, if significant error overshoots are to be avoided in frequencies not appearing in Eqs (21a), (22a). For the other 2 methods, the "rigid body frequency area" B is chosen equal to zero. In all cases the lower limit for the suppression of residual vibrations is $\omega_{\mathrm{C}}=1 \mathrm{rad} / \mathrm{s}$. The effect of increasing the number of the necessary coefficients $N$ is a drastic reduction of the error in the high frequency range above $\omega=2 \mathrm{rad} / \mathrm{s}$ and a minor improvement in the area between $\omega=1 \mathrm{rad} / \mathrm{s}$ and $\omega=2 \mathrm{rad} / \mathrm{s}$. The improvement 


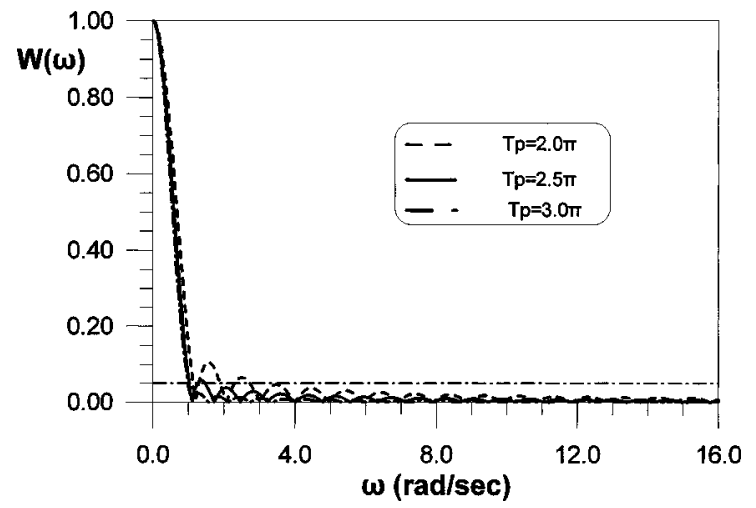

Fig. 5. Effect of the total pulse duration time $T_{p}$ on the error function of Fourier I method $(N=64)$.

using more coefficients than $N=64$ is marginal, unless a higher robust area above $\omega_{\max }$ of Eq. (20a) is to be achieved. Also, above $N=64$, all the results of the corresponding methods belonging in Case I and II become practically identical.

Comparing the individual behavior of each of the three proposed methods, the Fourier method leads to the best results, while the Chebyshev method is quite close. However, although the global solution approaches (Fourier, Chebyshev) indicate overall a better behavior than the Direct Solution Approach, only this last method can lead to the exact cancellation of the residual vibration error at well specified frequencies.

Then, the effect of increasing the total pulse duration time $T_{p}$ is examined for a total impulse number of $N=64$. The results for $T_{p}=2 \pi, 2.5 \pi, 3 \pi \mathrm{s}$ for the Fourier and the Chebyshev methods are shown respectively in Figs 5 and 6. The effect of increasing the total pulse duration time $T_{p}$ is the significant decrease of the residual vibration error in the whole frequency range, with more drastic effect in the area between $\omega=1 \mathrm{rad} / \mathrm{s}$ and $\omega=2 \mathrm{rad} / \mathrm{s}$.

Comparing the individual behavior of each of the two methods, the Fourier method leads to the best results.

The major conclusion from the above results (Fig. 5), is that the Fourier method can lead to the practical cancellation of the residual vibration error (error value below $5 \%$ ) for the whole frequency range above a specific frequency $\omega_{\mathrm{C}}$, when the impulse duration time is chosen as:

$$
\begin{aligned}
& T_{p}=(5 / 4) T_{\mathrm{C}}, \\
& T_{\mathrm{C}}=2 \pi / \omega_{\mathrm{C}} .
\end{aligned}
$$

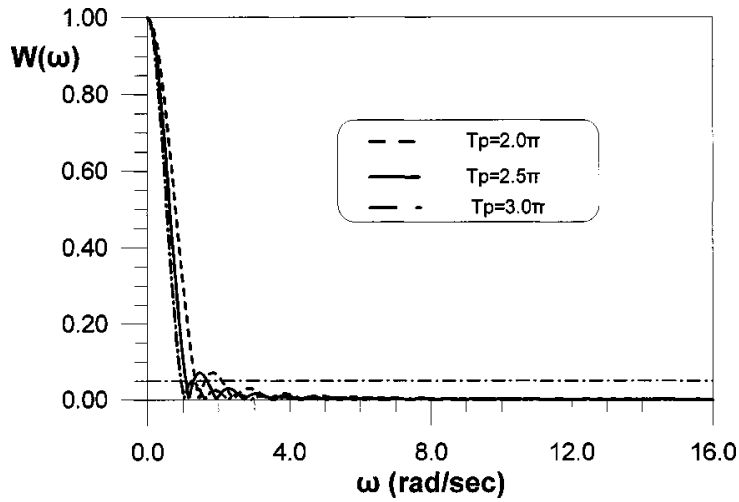

Fig. 6. Effect of the total pulse duration time $T_{p}$ on the error function of Chebyshev I method $(N=64)$

The corresponding pulse sequences in this case are shown in Fig. 7 for $N=4,16,64$ coefficients.

\section{Numerical experiments}

The results of the design procedure of Section 2 are then applied to an SDOF system, shown in Fig. 8. The system includes several non-linearities, in order to test the robustness of the proposed methods to variations of a broad range of system parameters. The equations of motion are:

$$
\begin{aligned}
\ddot{x}+ & 2 \zeta \omega(\dot{x}-\dot{\nu})+\left(\omega^{2}+k_{n}\right) \\
& \times(x-\nu)+F_{D}=0 \\
\omega= & \sqrt{k / m} \\
\zeta= & c / 2 m \omega \\
k_{n}= & D_{n}(x-\nu)^{2} \\
F_{D}= & C_{d} \dot{x}|\dot{x}| .
\end{aligned}
$$

The original guidance function is a ramp with a rise time of $5 \mathrm{~s}$. This function is shown in Fig. 9, together with the conditioned guidance function, derived according to Eq. (6) with the application of the impulse sequence of Fig. 7(c). Typical response curves of the original and the conditioned system, corresponding to $\omega=1 \mathrm{rad} / \mathrm{s}$, are shown in Fig. 10 .

First, the introduction of a PID controller is considered. An extensive treatment of the effect of PID controllers on flexible mechanical systems can be found in [3]. The $P$ term results in "stiffening" the mechanical system, increasing thus the natural frequency. The $D$ term results in adding damping to the system, re- 


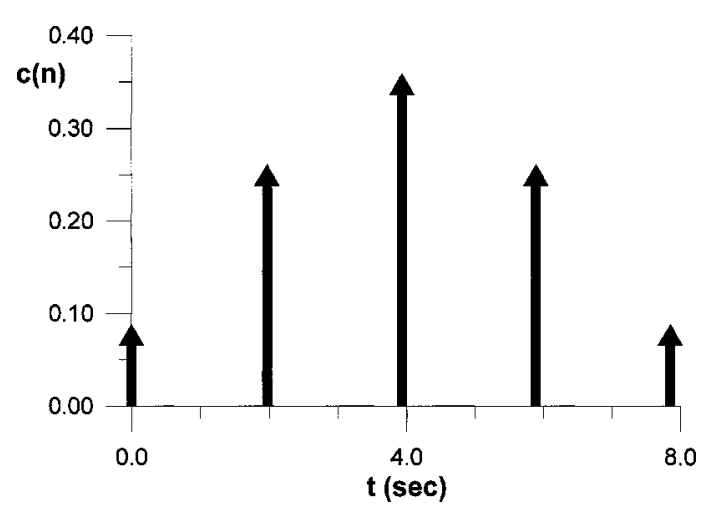

(a)

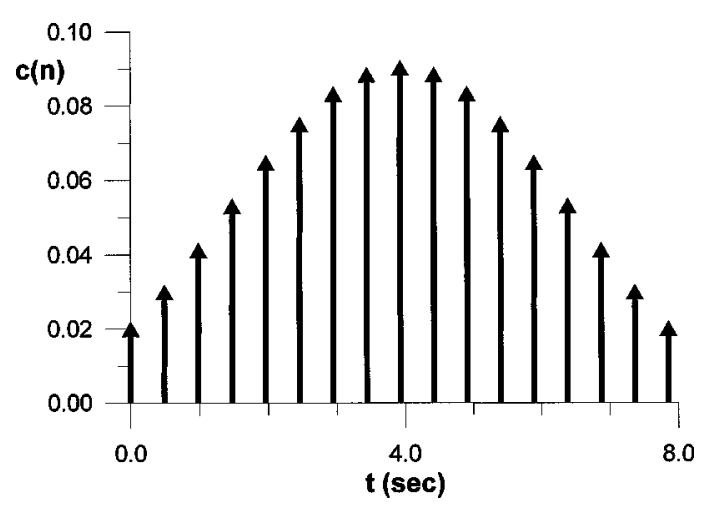

(b)

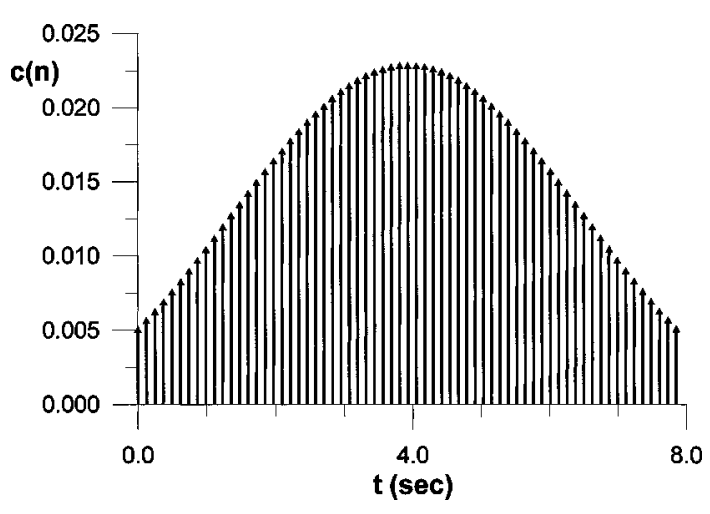

(c)

Fig. 7. Fourier I impulse sequences for $T_{p}=2.5 \pi$. (a) $N=4$, (b) $N=16$, (c) $N=64$.

sulting however to abrupt control actions. Based on the above guidelines, a PID controller is designed for the undamped system with $\omega=1 \mathrm{rad} / \mathrm{s}$. It provides a guidance function $u(t)=u_{\mathrm{C}}(t)$, tracking the error between the original ramp function and the system response $x(t)$. The $P$ term is chosen equal to 15 , result-

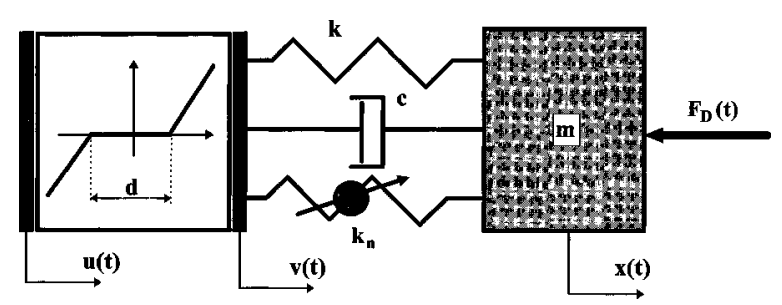

Fig. 8. Dynamic system, used in the numerical experiments.

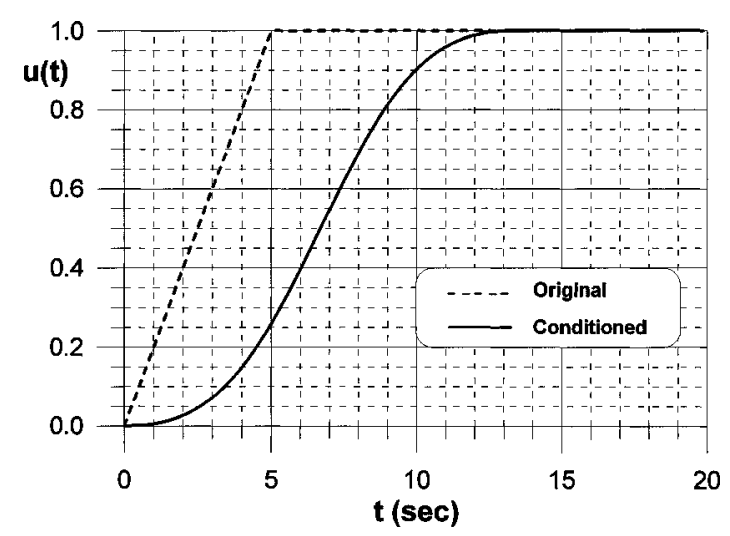

Fig. 9. Original and conditioned guidance function.

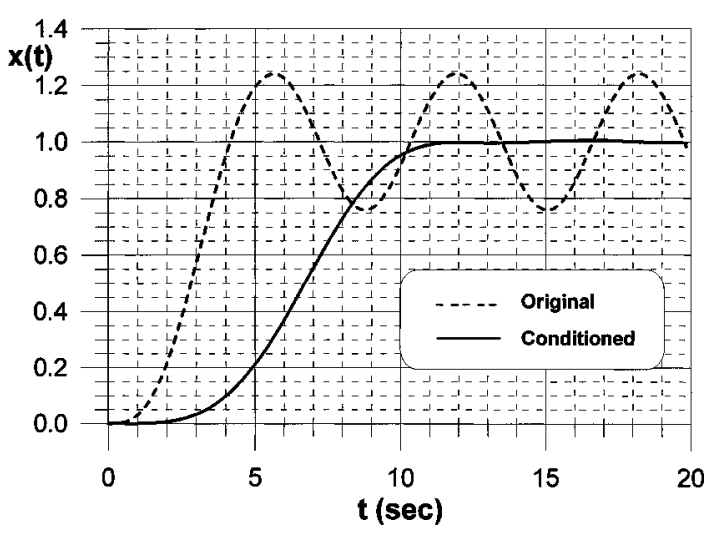

Fig. 10. System response to the original and conditioned guidance functions ( $\omega=1 \mathrm{rad} / \mathrm{s}$, all other variables set equal to zero).

ing to a system with a new eigenperiod of $4 \mathrm{rad} / \mathrm{s}$. The $D$ term is chosen equal to 5.656 , resulting to a new damping ratio of 0.707 .

Initially, no limits were applied to this PID controller (unlimited PID controller). The system response is shown in Fig. 11(a), indicating an almost perfect behavior. However, observing in Fig. 11(b) the elastic force on the spring introduced by this type of control, the penalty paid can be easily realized. Apart from actuator saturation problems, abrupt and significant elastic forces are present. 


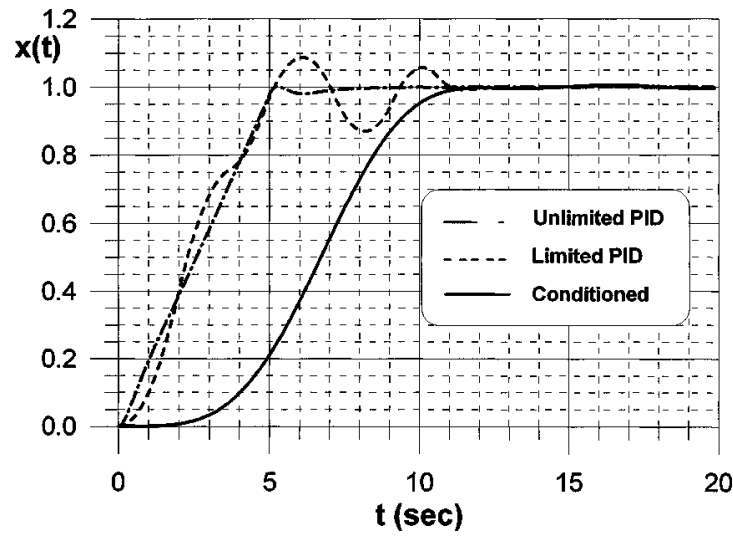

(a)

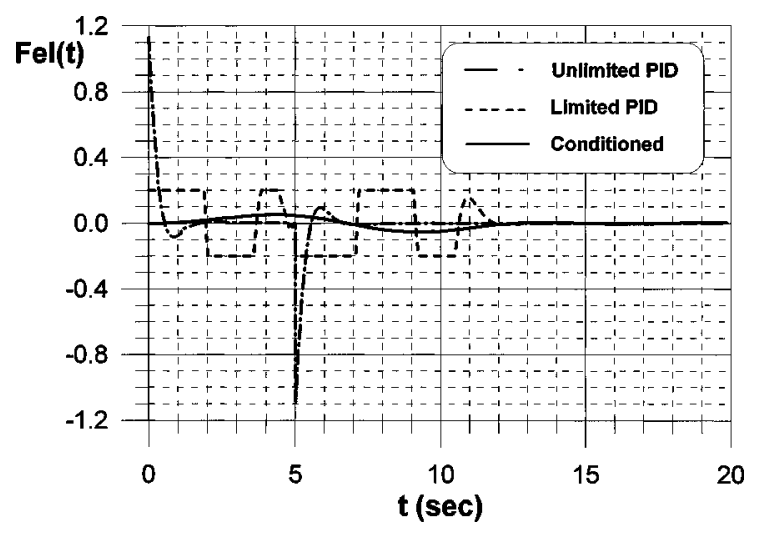

(b)

Fig. 11. System response to the unlimited and limited PID control ( $\omega=1 \mathrm{rad} / \mathrm{s}$, all other variables set equal to zero). (a) System response, (b) elastic force on the spring.

For this reason, the controller was limited (limited PID controller), so that the elastic force on the spring cannot exceed a value of 4 times the maximum spring force, induced by the guidance method of this paper. The results indicate a drastic decrease of the performance of the PID controller. Although the system response reaches the final value at the same time as that by the guidance function, it exhibits significant overshootings and elastic forces.

Then the effect of the variation of several parameters to the residual vibration error function is considered. For the comparison of the results, a total residual error function at time $T_{\mathrm{e}}=20 \mathrm{~s}$ is defined as:

$$
\operatorname{Er}\left(T_{\mathrm{e}}\right)=\left\{\left[x\left(T_{\mathrm{e}}\right)-\nu\left(T_{\mathrm{e}}\right)\right]^{2}+\left[\dot{x}\left(T_{\mathrm{e}}\right) / \omega\right]^{2}\right\}^{1 / 2} .
$$

The system natural frequency is varied over a range of $\omega=1-4 \mathrm{rad} / \mathrm{s}$, with all the other parameters set equal

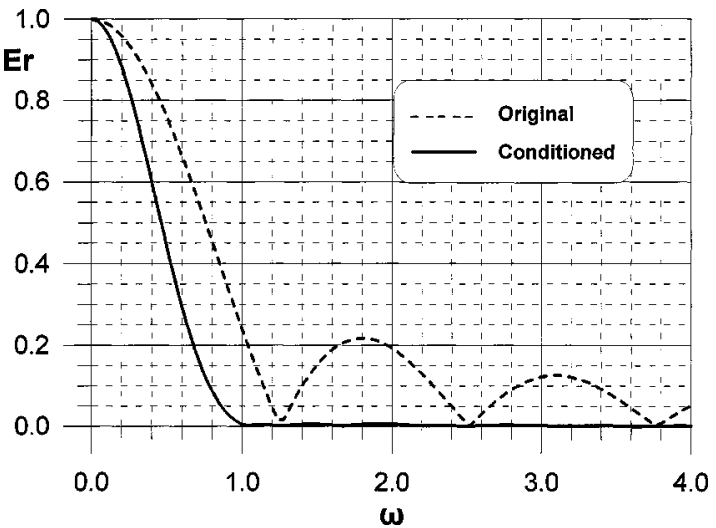

Fig. 12. Residual error function for variations of the eigenfrequency $\omega$.

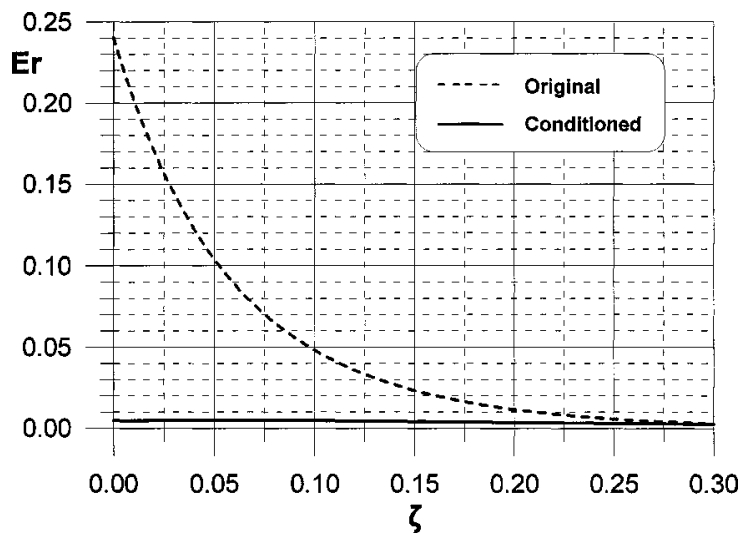

Fig. 13. Residual error function for variations of the damping ratio $\zeta$.

to zero. The corresponding results are shown in Fig. 12. The conditioned guidance function leads to zero vibration error over the whole specified frequency range, contrary to the original guidance.

Further system parameters were subsequently varied. In all cases, the natural frequency is set to $\omega=$ $1 \mathrm{rad} / \mathrm{s}$ and all the other non further mentioned parameters are set equal to zero. The damping ratio is varied over a range of $\zeta=0$ to $\zeta=0.30$. The corresponding error is shown in Fig. 13. As already mentioned in Section 2, the effect of damping is positive to the reduction of the residual vibration, as shown by its effect to the response at the original guidance function. However, Fig. 13 clearly verifies the assumption used in Section 2 for the derivation of Eq. (10) from Eq. (9): The design of a robust method with respect to natural frequency variations ensures also robustness for damping ratio variations. 


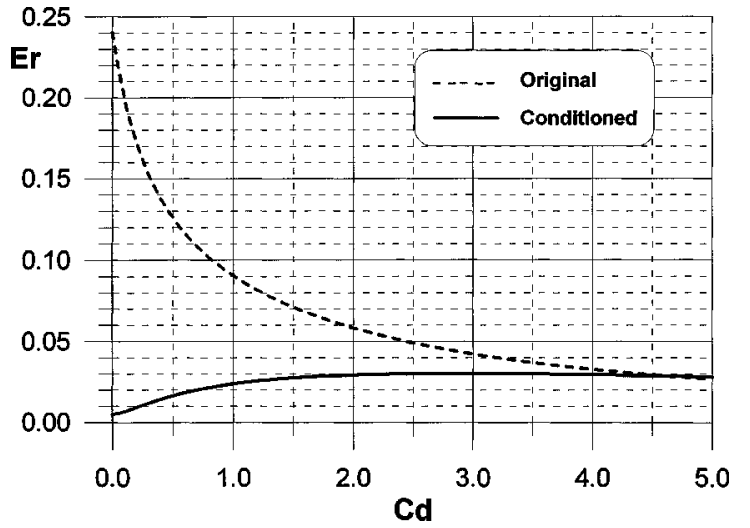

Fig. 14. Residual Error function for variations of the drag force coefficient $C_{d}$.

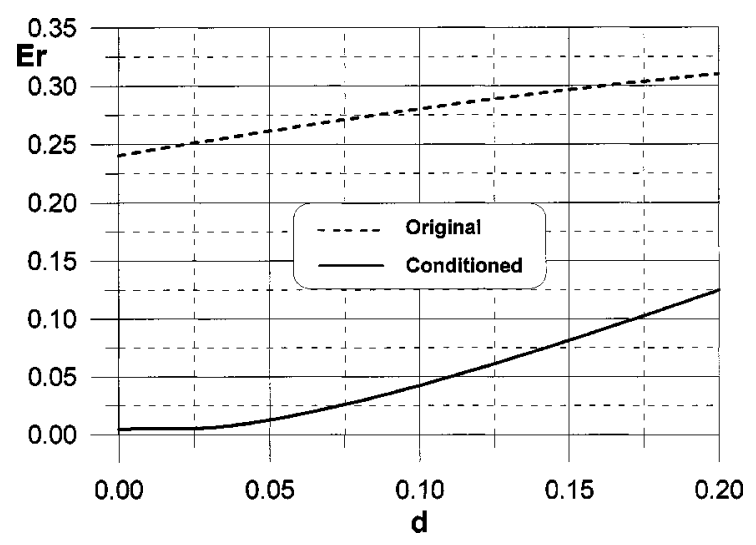

Fig. 15. Residual error function for variations of the dead band zone $d$.

The purpose of the numerical experiments further shown, is to check the validity of the above assumption also for variations of the other nonlinear system parameters.

Figure 14 presents the error for variations of the drag coefficient in the range $C_{d}=0$ to 5 . As expected due to the damping nature of the drag force, the effect is positive for the original guidance function. A marginally negative effect is observed for the conditioned guidance function. However, even in this case, the residual error still remains below the accepted value of $5 \%$, indicating a robust behavior.

Figure 15 presents the error for variations of the dead-band zone width in the range $d=0$ to 0.20 . The effect of this type of linearity is to increase the residual vibration error in a similar amount for both types of excitation. However, the error for the conditioned guidance function is significantly lower than that of the unconditioned guidance.

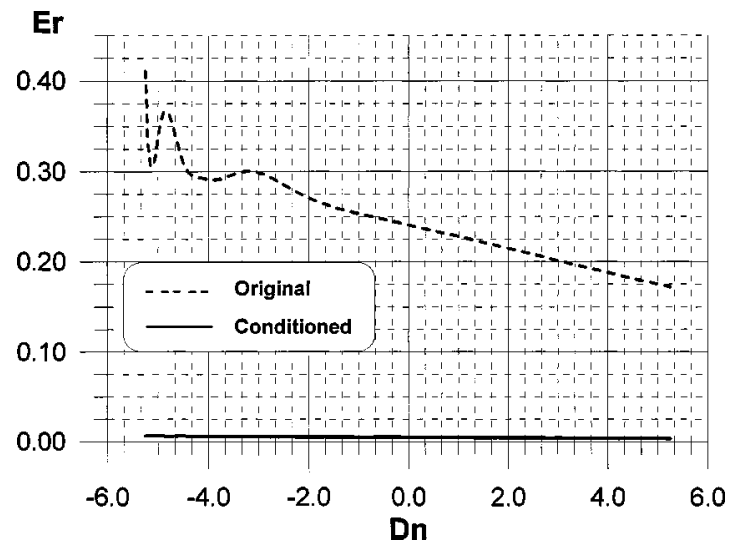

Fig. 16. Residual error function for variations of the non-linear spring constant $D_{n}$.

In both cases of drag force and dead-band zone nonlinearities, residual vibrations increase for the conditioned guidance function, due to the abrupt changes that are produced to its shape by non-linearities of this form. Thus, in these cases, some further research is needed, in order to enhance the frequency robustness properties of the guidance function with additional features, such as the one proposed in [8].

Finally, the effect of variations of the non-linear spring constant are considered. Figure 16 presents the error for positive variations in the range $D_{n}=-5.25$ to +5.25 . The error of the unconditioned guidance is significant, increasing with negative values of $D_{n}$. Furthermore, the system response to the original guidance for values of $D_{n}<-5.3$ becomes unstable. The conditioned guidance function performs quite robustly, similarly to the linear case.

\section{Conclusion}

Preconditioning any guidance function with a series of impulses designed according to the approach developed in this paper, can lead to drastic residual vibration suppression. When the total duration time of the impulse sequence is set to a value larger than 1.25 times the maximum expected natural period, the residual vibration error can be practically suppressed over the whole frequency range. This robustness property of the impulse sequence is additionally valid in practice, not only for variations of other linear system parameters (e.g., damping ratio), but also for variations of a number of non-linear parameters.

The practical implementation of the method requires just a summation in the form of Eq. (6c). This oper- 
ation can be performed either online or off-line, quite independently from the type of the original guidance. Thus, the method can be easily applied in practice to any mechanical system, with any form of original guidance, either derived mathematically, or input directly to the system.

\section{References}

[1] I. Antoniadis and A. Kanarachos, A spline-based guidance with enhanced frequency robustness for the motion of flexible part handling manipulators, Mechatronics 6 (1996), 761-777.

[2] D.M. Aspinwall, Acceleration profiles for minimizing residual response, ASME J. of Dynamic Systems, Measurement and Control 102 (1980), 3-6.

[3] T.D. Burton, Introduction to Dynamic System Analysis, McGraw-Hill, NJ, 1994.

[4] S.P. Bhat, M. Tanaka and D.K. Miu, Experiments on point-topoint position control of a flexible beam using Laplace transform technique - Part I: Open-loop, ASME J. of Dynamic Systems, Measurement and Control 113 (1991), 432-437.

[5] R.L. Farrenkopf, Optimal open-loop maneuver profiles for flexible spacecraft, J. of Guidance and Control 2(6) (1979), 491498.

[6] A.A. Goldenberg and F. Rakhsa, Feedforward control of a single-link flexible robot, Mechanism and Machine Theory 21(4) (1986), 325-335.
[7] A. Kanarachos and T. Costopoulos, Optimierung von Bewegungsbahnen, Werkstattstechnik 77 (1987), 45-49.

[8] A. Kanarachos, M. Sfantsikopoulos and P. Vionis, A splinesbased control method for robot manipulators, Robotica 7 (1988), 213-221.

[9] A. Kanarachos and I. Antoniadis, Machine Dynamics, Papasotoiriou, Athens, 1998 (in Greek).

[10] P.H. Meckl and W.P. Seering, Reducing residual vibration in systems with time varying resonances, in: Proc. of the 1987 IEEE Int. Conf. on Robotics and Automation, pp. 1690-1695.

[11] W.H. Press, S.A. Teukolsky, W.T. Vetterling and B.P. Flannery, Numerical Recipes in FORTRAN, Cambridge Univ. Press, 2nd edn, 1992.

[12] O. Sato, H. Shimojima and T. Kaneko, Positioning control of a gear train system including flexible shafts, JSME Int. J. 30 (1987), 1465-1472.

[13] N.C. Singer and W.P. Seering, Preshaping command inputs to reduce system vibration, ASME J. of Dynamic Systems, Measurement and Control 112 (1990), 76-82.

[14] T. Singh and G.R. Heppler, Shaped input control of a system with multiple modes, ASME J. of Dynamic Systems, Measurement and Control 115 (1993), 341-347.

[15] T. Singh and S.R.Vadali, Robust time-delay control of multimode systems, Int. J. of Control 62 (1995), 1319-1339.

[16] W.E. Singhose, L.J. Porter, T.D. Tuttle and N.C. Singer, Vibration reduction using multi-hump input shapers, ASME J. of Dynamic Systems, Measurement and Control 112 (1997), 76-82.

[17] O.J.M. Smith, Feedback Control Systems, McGraw-Hill, New York, 1958. 

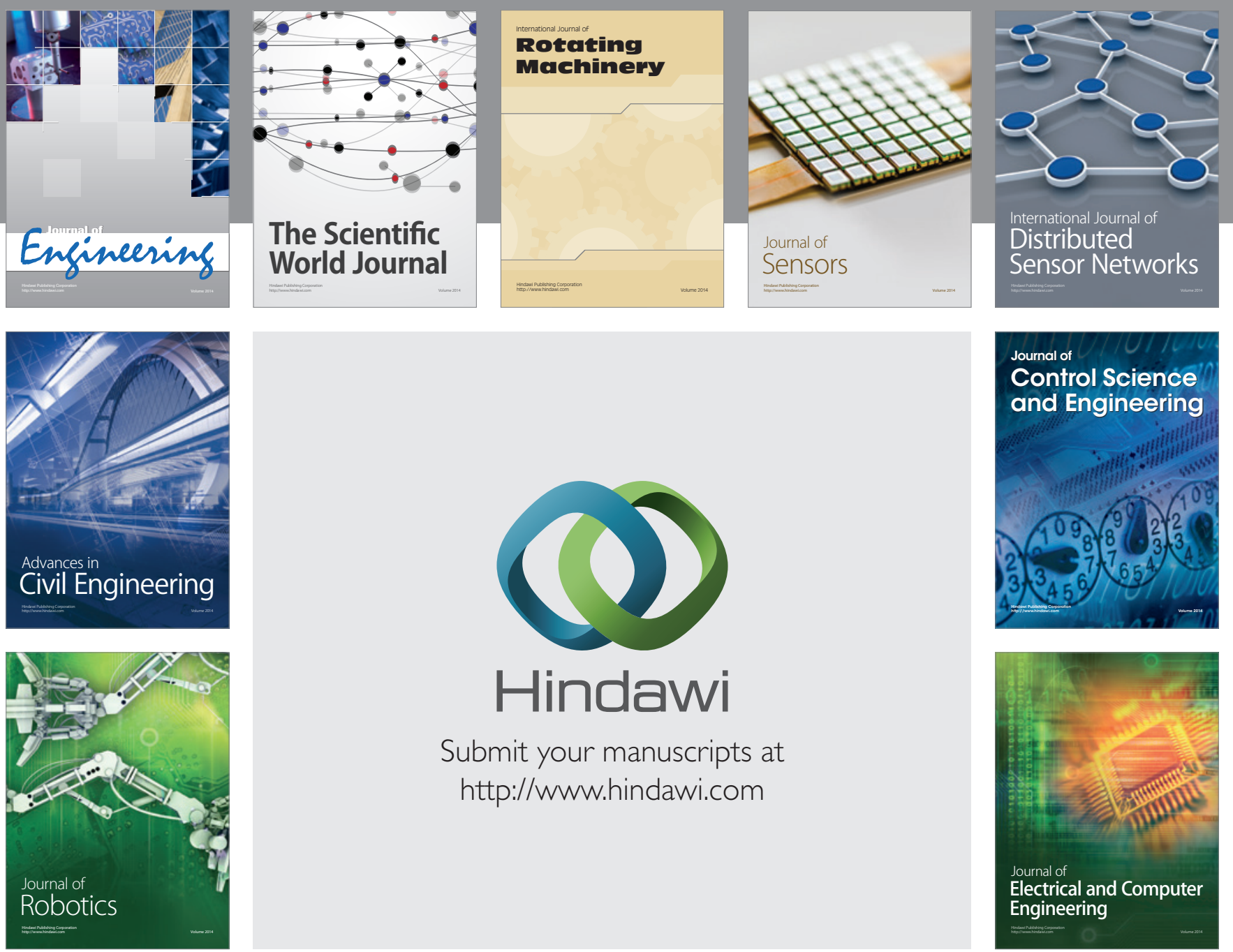

Submit your manuscripts at

http://www.hindawi.com
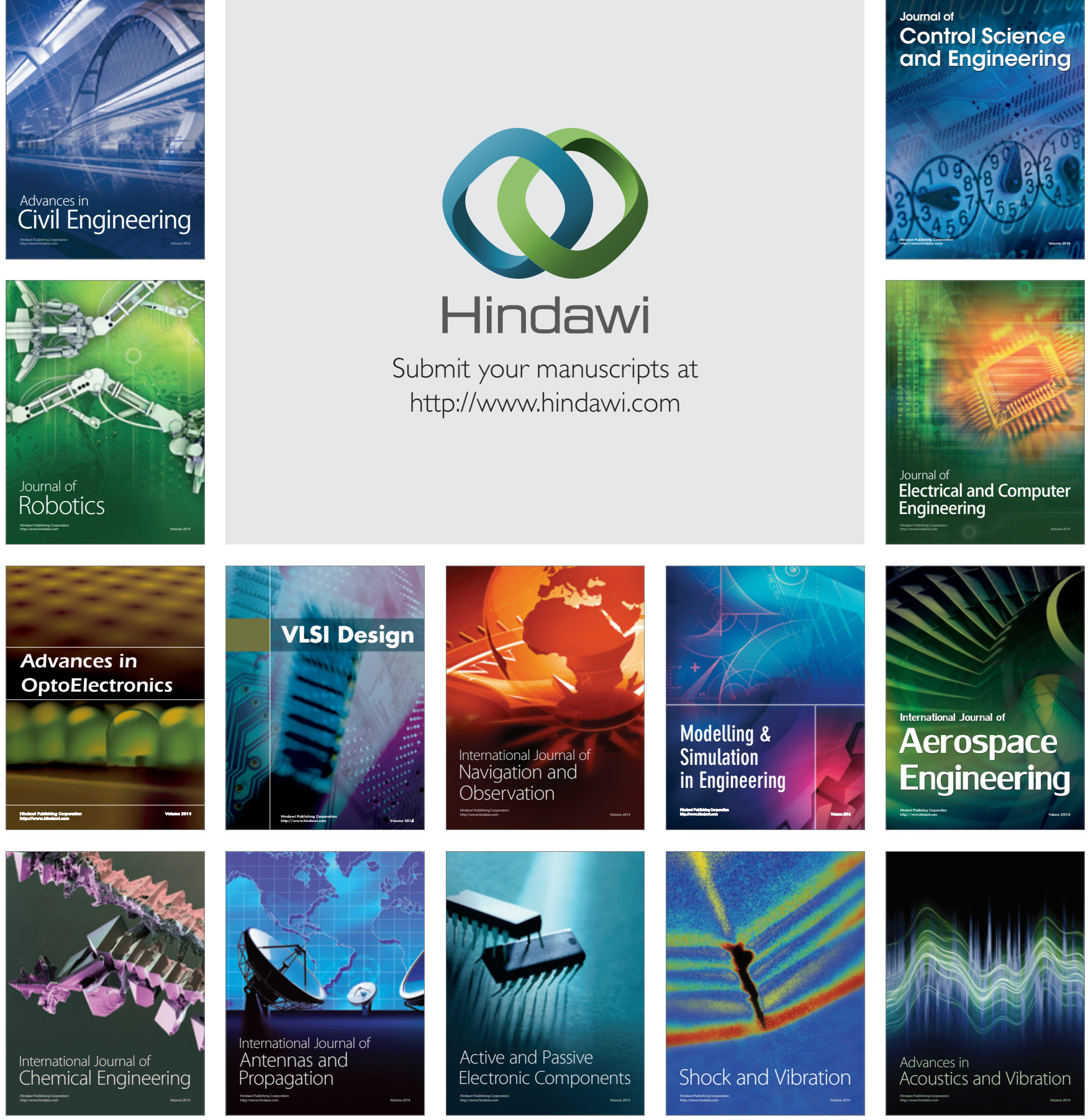